\title{
Nanoscale dielectric microscopy of non-planar samples by lift- mode electrostatic force microscopy
}

\author{
M Van Der Hofstadt ${ }^{1,2}$, R Fabregas ${ }^{1}$, M C Biagi ${ }^{1}$, L Fumagalli $^{3}$ and G Gomila*,1,2 \\ ${ }^{1}$ Institut de Bioenginyeria de Catalunya (IBEC), c/ Baldiri i Reixac 11-15, 08028, Barcelona, Spain \\ ${ }^{2}$ Departament d'Enginyeries: Electrònica, Universitat de Barcelona, C/ Martí i Franqués 1, 08028, \\ Barcelona, Spain \\ ${ }^{3}$ School of Physics and Astronomy, University of Manchester, Oxford Road, Manchester, M13 9PL, United \\ Kingdom
}

*Corresponding Author: ggomila@ibecbarcelona.eu

\begin{abstract}
Lift-mode Electrostatic Force Microscopy (EFM) is one of the most convenient imaging modes to study the local dielectric properties of non-planar samples. Here we present the quantitative analysis of this imaging mode. We introduce a method to quantify and subtract the topographic crosstalk from the lift-mode EFM images, and a 3D numerical approach that allows extracting the local dielectric constant with nanoscale spatial resolution free from topographic artifacts. We demonstrate this procedure by measuring the dielectric properties of micropatterned $\mathrm{SiO}_{2}$ pillars and of single bacteria cells, thus illustrating the wide applicability of our approach from materials science to biology.
\end{abstract}

Index Terms-2. Nanotechnology: AFM, dielectric constant, EFM, dielectrics, non-planar surfaces.

\section{Introduction}

In recent years a number of scanned probe microscopy techniques sensitive to the local dielectric permittivity properties of dielectric materials have been developed. Among them, we can cite nanoscale capacitance microscopy [1-3], electrostatic force microscopy (EFM) [4-10], nanoscale impedance microscopy [11,12], scanning polarization force microscopy [13-16], scanning microwave microscopy (SMM) $[17,18]$ and nanoscale non-linear dielectric microscopy [19]. These techniques have allowed measuring the electric permittivity with nanoscale spatial resolution on planar samples, such as thin oxides, polymer films and supported biomembranes $[2-4,8,10]$, and on non-planar ones, such as, single carbon nanotubes, nanowires, nanoparticles, viruses and bacterial cells [20-30].

Despite these successful applications, using these techniques to study highly non-planar samples remains difficult in some cases. In particular, it is still a challenge to obtain the local dielectric permittivity properties of samples with large topographic variations in the range of hundreds of nanometers. The reason being that for these samples, measurements are taken with the tip following the sample surface topography (in contact, intermittent contact or by two pass modes, such as, 'lift' mode). As a consequence, the tip-substrate distance varies during the image acquisition, thus inducing spurious capacitance variations that are not related to the dielectric properties of the sample. That is, dielectric images of non-planar samples can be severely affected by topographic crosstalks [31-34].

Very recently, we have proposed a simple method to address this problem in the framework of scanning microwave microscopy [28]. It consists in reconstructing a dielectric topographic crosstalk image from the measured sample topography and a measured dielectric approach curve taken on the bare part of the substrate. By removing the crosstalk image from the measured dielectric image, one obtains an intrinsic dielectric image, which is closely related to the local dielectric permittivity properties of the sample, and which is directly amenable to a quantitative analysis with the help of finite-element numerical calculations.

Here, we generalize this approach to the case of lift mode Electrostatic Force Microscopy (EFM). We present the procedure to construct EFM topographic crosstalk images, to further generate intrinsic EFM images, which can then be directly interpreted in terms of the local polarization properties of the sample. Quantification of the resulting intrinsic EFM images is carried out by means of full 3D image numerical calculations. The procedure is demonstrated here on micropatterned $\mathrm{SiO}_{2}$ pillars and to 
a single bacterial cell, in order to show the wide range of possible applications.

\section{Topographic crosstalk in lift-mode EFM}

Let us consider the typical case of lift-mode EFM images acquired in amplitude detection mode (a similar procedures can be applied to other EFM detection modes, such as frequency shift detection). In this two pass technique, in the first pass the topography of the sample is recorded in any AFM imaging mode, and in the second pass the EFM signal is recorded by lifting the tip at a fixed distance, $z_{\text {lift }}$, from the sample surface. The EFM signal is obtained by applying an ac voltage of amplitude $v_{\omega}$ and frequency $\omega$ (far from the mechanical resonance frequency of the cantilever) on the conductive probe and recording the cantilever oscillation amplitude at frequency $2 \omega, A_{2 \omega}$. The oscillation amplitude is then related to the tip-sample capacitance gradient, $d C / d z$, through the well-known relationship

$$
\frac{d C}{d z}=\frac{4}{v_{\omega}^{2}} k A_{2 \omega}
$$

where $k$ is the equivalent spring constant of the cantilever. From the capacitance gradient, quantitative information on the local dielectric permittivity properties of the sample can be obtained with the help of the appropriate theoretical model. A schematic representation of liftmode EFM, with the definition of the more relevant parameters, is shown in figure 1 . Note that the lift path $z(\vec{x})=h(\vec{x})+z_{\text {lift }}$ follows the measured topography, $h(\vec{x})$, where $\vec{x}=(x, y)$ represents a position on the plane of the sample. In general, however, the measured topography is affected by tip-sample convolution effects

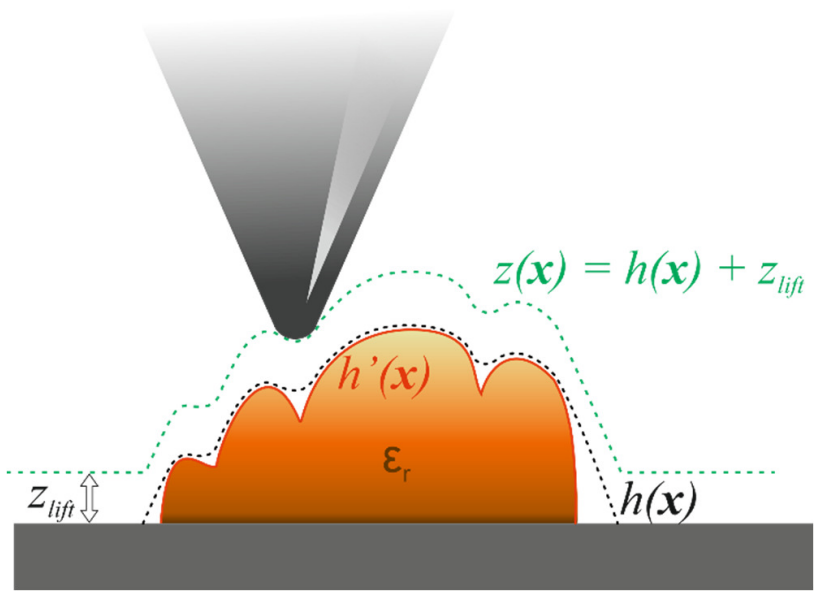

Figure 1. Schematic representation of the lift mode imaging with the definition of the main parameters. and may differ from the actual sample surface topography, $h^{\prime}(\vec{x})$. For highly non-planar samples, tip convolution effects can be significant and they have to be taken into account.

Since electrostatic interactions are long ranged, the tip interacts, in general, with both the substrate and the sample. As a result, variations in tip-substrate distance occurring during lift mode imaging induce some capacitance gradient variations that also contribute to the capacitance gradient image. These capacitance gradient contributions, which we refer to as topographic crosstalk contribution, are independent from the dielectric permittivity properties of the sample and, hence, would be present even if the sample showed no electric polarizability (or the sample was not present). If $C^{\prime}\left(\vec{x}, z ; \varepsilon_{r}\right)$ is the measured capacitance gradient for a sample with dielectric constant, $\mathcal{E}_{r}$, when the tip is located at a distance $z$ from the substrate at position $\vec{x}=(x, y)$, then the capacitance gradient topographic crosstalk at lift distance $z_{\text {lift }}$ is given by

$$
C_{\text {cross }}^{\prime}\left(\vec{x}, z_{\text {lift }}\right)=C^{\prime}\left(\vec{x}, h(\vec{x})+z_{\text {lift }} ; \varepsilon_{r}=1\right)
$$

By definition, the crosstalk contribution is a function of the lift distance $z_{\text {lift }}$ and the measured topography, $h(\vec{x})$, as well as, of the probe dimensions.

Given the topographic crosstalk contribution, we can subtract it from the measured capacitance gradient image to obtain, what we call, the intrinsic capacitance gradient image, $C_{\text {int }}^{\prime}\left(\vec{x}, z_{\text {lift }} ; \varepsilon_{r}\right)$, given by,

$$
\begin{gathered}
C_{\text {int }}\left(\vec{x}, z_{l i f f} ; \varepsilon_{r}\right) \equiv C\left(\vec{x}, h(\vec{x})+z_{\text {lift }} ; \varepsilon_{r}\right)-C_{\text {cross }}\left(\vec{x}, z_{\text {liff }}\right) \\
\quad=C\left(\vec{x}, h(\vec{x})+z_{\text {liff }} ; \varepsilon_{r}\right)-C\left(\vec{x}, h(\vec{x})+z_{\text {liff }} ; \varepsilon_{r}=1\right)
\end{gathered}
$$

The intrinsic capacitance gradient image shows two useful properties, namely, it is different from zero on those parts of the image where the sample is present, and it is free from stray capacitance contributions (e.g. nonlocal cantilever contributions), since these contributions are subtracted. It should be noted, that the intrinsic capacitance gradient is still dependent on the sample geometry and dimensions since they determine the overall electric polarization of the sample, and hence, the force acting on the tip [31-34].

Experimentally the topographic crosstalk capacitance gradient image can be constructed following the procedure that we applied for the case of capacitance measurements in Ref. [28]. Briefly, one measures a 
(a)

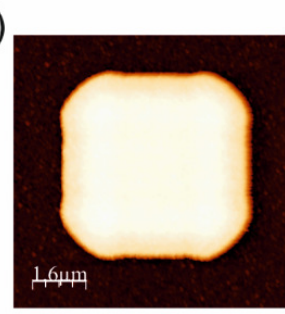

(b)

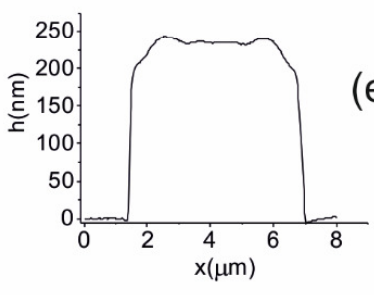

(d)

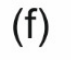

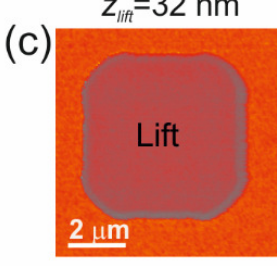
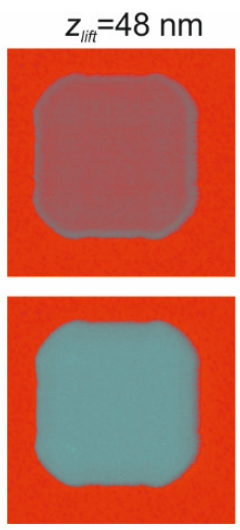

(e)
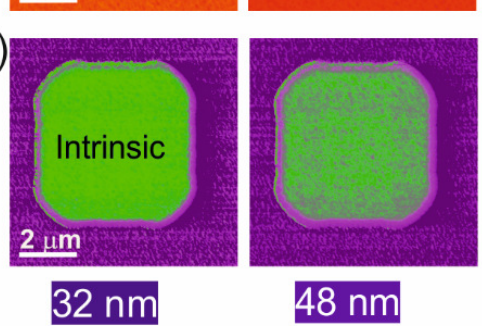
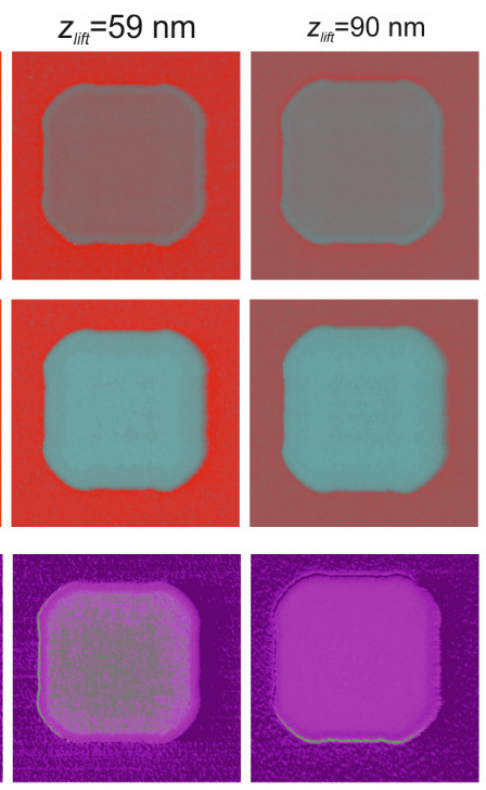

$59 \mathrm{~nm}$
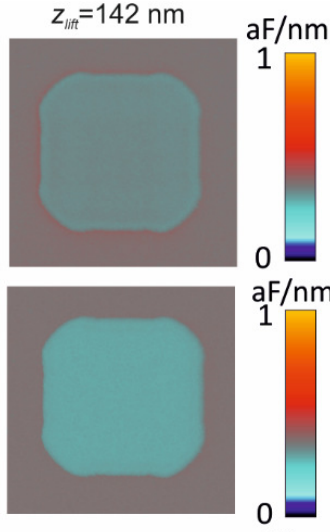

$\mathrm{aF} / \mathrm{nm}$

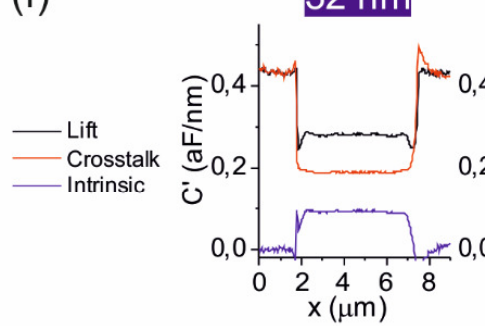

(g)
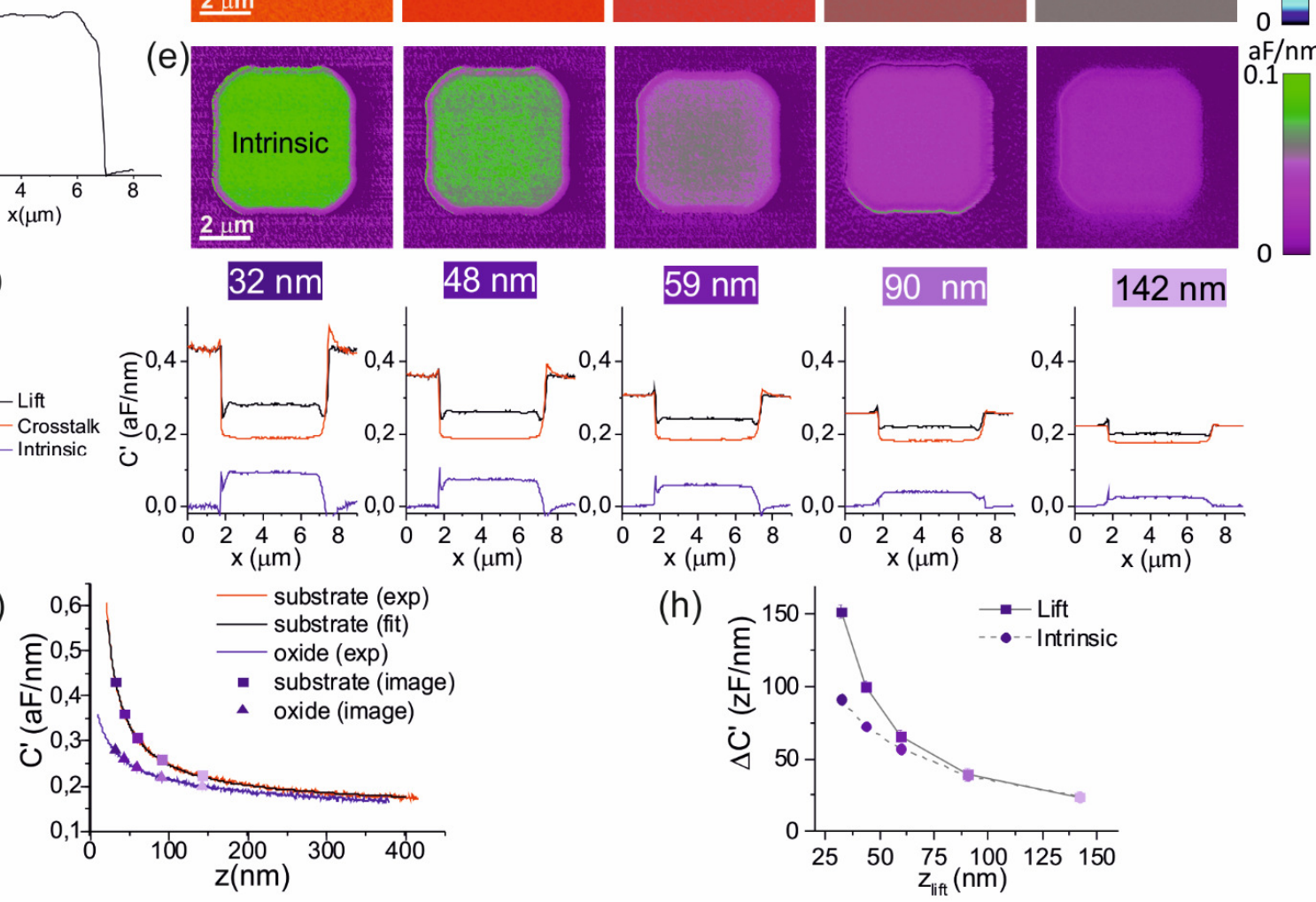

$\mathrm{aF} / \mathrm{nm}$

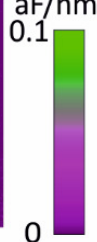

$142 \mathrm{~nm}$
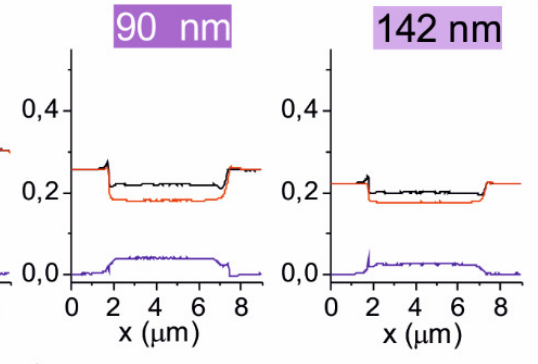

(h)

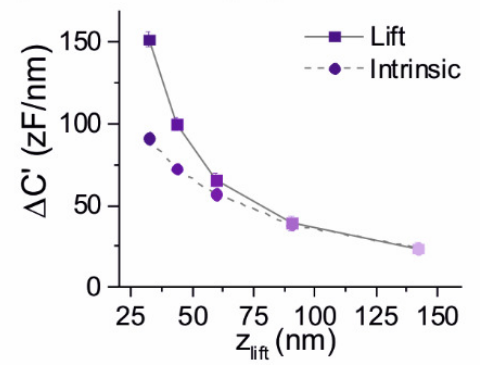

Figure 2. (a) Topographic image of a micropatterned $\mathrm{SiO}_{2}$ pillar of height $\sim 236 \mathrm{~nm}$ on a highly doped silicon substrate. (b) Topographic cross-section profile along the center in (a). (c) Series of five $2 \omega$-capacitance gradient images obtained in lift mode at lift distances of $32 \mathrm{~nm}, 48 \mathrm{~nm}, 59 \mathrm{~nm}, 90 \mathrm{~nm}$ and $142 \mathrm{~nm}$, respectively. Note that absolute capacitance gradient values are shown. (d) Series of five topographic crosstalk capacitance gradient images reconstructed with the capacitance gradient approach curve measured on the substrate and shown in (g), red line, and the topographic image in (a), for each lift distance. Absolute values are shown. (e) Series of five intrinsic capacitance gradient images obtained from the subtraction of the capacitance gradient topographic crosstalk images in (d) from the measured capacitance gradient images in (c). (f) Series of five sets of cross-section profiles along the center of the images in (c) (black lines), (d) (red lines) and (e) (blue lines). (g) (Symbols) Absolute capacitance gradient values obtained from the images on the substrate (squares) and on the center of the oxide (triangles) as a function of the lift distance. (Continuous lines) Measured capacitance gradient approach curves measured on the substrate (red line) and on the center of the oxide (blue line). The black line is a least square fitting of the theoretical model to the approach curve on the substrate to calibrate the probe geometry giving a tip radius $R=166 \pm 2 \mathrm{~nm}$ and a half cone angle $\theta=15.0 \pm 0.5^{\circ}$ (see section 3 ). The theoretical curves are shifted by a fitted constant capacitance gradient offset of $k_{\text {stray }}=0.128 \pm 0.002 \mathrm{aF} / \mathrm{nm}$ to account for stray effects associated to the cantilever and not included in the model. (h) Capacitance gradient contrast in the lift (square symbols) and intrinsic (circle symbols) images shown in (c) and (e), respectively, as a function of the lift distance (the lines are guides to the eyes).

capacitance gradient approach curve on a bare part of the substrate, $C_{\text {subs }}^{\prime}(z)$, and then substitutes in it the tipsubstrate distance, $z$, by the tip substrate distance during the lift imaging, $z_{\text {lift }}+h(\vec{x})$, thus giving the topographic crosstalk capacitance gradient image, i.e. $C_{\text {cross }}^{\prime}\left(\vec{x}, z_{\text {lift }}\right)=C_{\text {subs }}^{\prime}\left(z_{\text {lift }}+h(\vec{x})\right)$.

We illustrate these concepts in Figure 2 for measurements obtained on a silicon dioxide pillar $\sim 236$ $\mathrm{nm}$ height and $\sim 5 \mu \mathrm{m}$ wide microfabricated on a highly 
doped silicon substrate (AMO Gmbh). The images have been obtained with a commercial AFM system (Nanotec Electronica, S.L.) coupled to an external lock-in amplifier (Anfatec Instruments AG), in lift-mode by applying a voltage of amplitude $3 \mathrm{~V}(\mathrm{rms})$ and frequency $2 \mathrm{kHz}$, and acquiring the cantilever oscillation amplitude at $4 \mathrm{kHz}$. CDT-CONTR doped diamond probes (Nanosensors) of spring constant $0.79 \mathrm{~N} / \mathrm{m}$ and nominal tip radius $\sim 100 \mathrm{~nm}$ have been used. Figure 2 a shows the topographic image of the $\mathrm{SiO}_{2}$ pillar, while figure $2 \mathrm{~b}$ shows a horizontal cross-section profile across the centre of the topographic image. A series of five lift-mode EFM capacitance gradient images obtained at lift distances $32 \mathrm{~nm}, 48 \mathrm{~nm}$, $59 \mathrm{~nm}, 90 \mathrm{~nm}$ and $142 \mathrm{~nm}$ are shown in figure 2c. Absolute values of the capacitance gradient are represented in the images. The precise value of the lift distance for each image, $z_{\text {lift, }}$ has been obtained by comparing the value of the capacitance gradient on a bare part of the substrate with the value obtained from an independently measured capacitance gradient approach curve on the substrate (square symbols and red line, respectively, in figure $2 \mathrm{~g}$ ). The EFM images are converted to capacitance gradient images by using Eq. (1).

For a given lift distance, the absolute values of the capacitance gradient (and hence of the electrostatic force acting on the tip) decrease when the tip moves from the substrate to the top of the pillar (see cross-section profiles in figure $2 \mathrm{f}$, black lines). We will show below that this decrease is strongly influenced by the fact that the probesubstrate distance increases when the tip moves away from the substrate to the top of the pillar (i.e. topographic crosstalk).

Moreover, when the lift distance increases, the absolute capacitance gradient values on both the substrate and the oxide decrease, as expected (see also cross-section profiles, black lines, in figure $2 \mathrm{f}$ ). This fact is explicitly shown in figure $2 \mathrm{~g}$ where we plot the capacitance gradient values on the substrate (square symbols) and on the oxide (triangle symbols) as a function of the lift distance (the error in the values is around $\sim 0.002 \mathrm{aF} / \mathrm{nm}$, smaller than the symbol). Instead, the values on the substrate (squares) overlap with the approach curve measured on it, since it is the condition to set the lift distances. The values obtained from the images on the oxide (triangles), once the lift distance has been adjusted with the substrate as mentioned, nicely overlap with an approach curve taken on the oxide (blue line in figure $2 \mathrm{~g}$ ) without no adjustment, thus confirming the stability of the measuring EFM set up and the equivalence of both types of measurements. It can be observed that the capacitance gradient values on the substrate (squares) decrease more rapidly than those on the oxide (triangles) when moving away from the substrate. This fact implies that the contrast in the images also decreases when increasing the lift distance, as it is explicitly shown in figure $2 \mathrm{~h}$ where we plot the lift contrast values (square symbols) as a function of lift distance. In particular, the contrast greatly decreases at lift distances larger than $\sim 100 \mathrm{~nm}$ in the present case.

With the help of the topographic image (figure 2a) and the approach curve measured on the substrate (red line in figure $2 \mathrm{~g}$ ), we have constructed the capacitance gradient topographic crosstalk images as explained above (equation 2). The results are shown in figure $2 \mathrm{~d}$, together with the cross-section profiles in figure $2 \mathrm{f}$ (red lines). These images show values and contrasts relatively similar to those of the lift images, thus confirming that topographic crosstalk contributes significantly to lift EFM images on highly non-planar samples. By subtracting the crosstalk images from the lift images (equation 3) we obtain the intrinsic capacitance gradient images (figure $2 \mathrm{e}$, note the change of range in the colour scale). As advanced before, the intrinsic capacitance gradient images show non null values on those parts of the image where there is the oxide, showing a positive contrast (see cross-section profiles in figure $2 \mathrm{f}$, blue lines). The contrast in the intrinsic capacitance gradient images decreases when the lift distance increases, as for the lift images, as shown in figure $2 \mathrm{~h}$ (circles). Note that, in absolute terms, the contrast in the intrinsic images is significantly smaller than that on the lift images, especially at short distances, (compare circles and squares in figure $2 \mathrm{~h}$ ), as a consequence of the subtraction of the topographic crosstalk contribution.

We note that the intrinsic capacitance gradient images also show non-null contrast values on positions corresponding to the substrate close to the oxide pillar. This non-null contrast is due to long range electrostatic lateral effects that are detected by the probe when still on the substrate but close to the oxide pillar. These lateral long range effects are identified in the lift mode image as an increase in the capacitance gradient signal with respect to the signal on the substrate when the tip approaches the oxide pillar at short lateral distances (below $\sim 200 \mathrm{~nm}$ in the present case). 


\section{Quantitative analysis of intrinsic capacitance gradient images}

Intrinsic capacitance gradient images provides a direct route to quantify the local electric permittivity of highly non-planar samples. First, it enables to directly visualize the homogeneity (or non-homogeneity) of the sample's dielectric properties not masked by topographic crosstalk artefacts. This allows one setting up theoretical models that already incorporate this information. For instance, in the case of the measurements performed on the oxide pillars a direct inspection of the intrinsic capacitance gradient images shown in figure $2 \mathrm{e}$ confirms the uniformity of the dielectric properties of the pillars, which can then be assumed in the theoretical model. Furthermore, the use of intrinsic capacitance gradient images also enables one to use simplified probe models. In particular, in the present case, in which the substrate can be assumed to be metallic from the modelling point of view (it can be approximated by a constant electric potential surface given the high doping density of silicon), the cantilever does not need to be included in the numerical calculations of the intrinsic capacitance gradient images. The reason being that for metallic substrates the cantilever contribution is included in both the lift EFM image and the reconstructed crosstalk image, and hence it is automatically subtracted and does not contribute to the intrinsic capacitance gradient image. Note, however, that in the case of thick insulator substrates, the microscopic parts of the probe, such as the cantilever, induce some indirect effects in addition to the direct stray effect mentioned above, and some contribution from them need to be included in the model $[29,35]$.

Based on this analysis, we calculate the intrinsic capacitance gradient images through the model system schematically depicted in figure $3 \mathrm{a}$. The tip is represented as a truncated cone of half-angle $\theta$, and cone height $H$, terminating in a tangent hemisphere of radius $R$ [35]. In addition, a disc of thickness $W$, overseeing the cone base by an amount $L$ is located onto the cone base to model eventual local cantilever effects. As explained above, given that the substrate is metallic-like we do not need to include these effects in the present work, so we take $L=$ $0 \mu \mathrm{m}$. The explicit tip geometry used in the calculations is determined by means of the tip calibration procedure described elsewhere $[8,26]$. Briefly, theoretical approach curves calculated for the tip on the bare substrate are least square fitted to an experimentally recorded approach curve on the metal, with the tip radius, $R$, and cone angle, $\theta$, as fitting parameters (other probe geometric parameters are fixed to nominal values: $H=12.5 \mu \mathrm{m}, W=3 \mu \mathrm{m}, L=0$ $\mu \mathrm{m})$. A constant offset, $k_{\text {stray }}$, is also included in the (a)

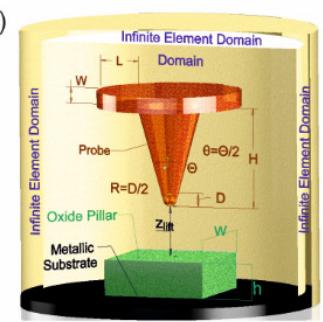

(c)

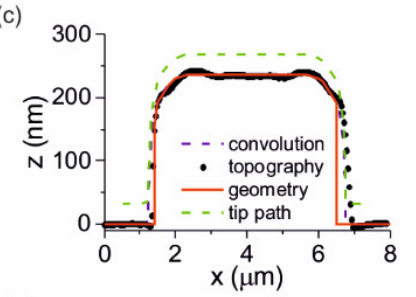

(e) Lift C' (zF/nm) $\quad 400^{(\mathrm{f})}$ Crosstalk C' (zF/nm)

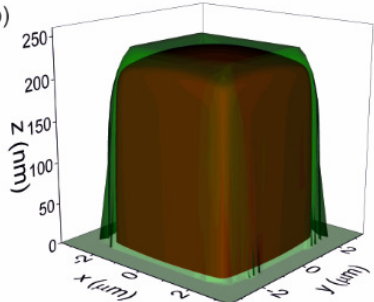

(d)
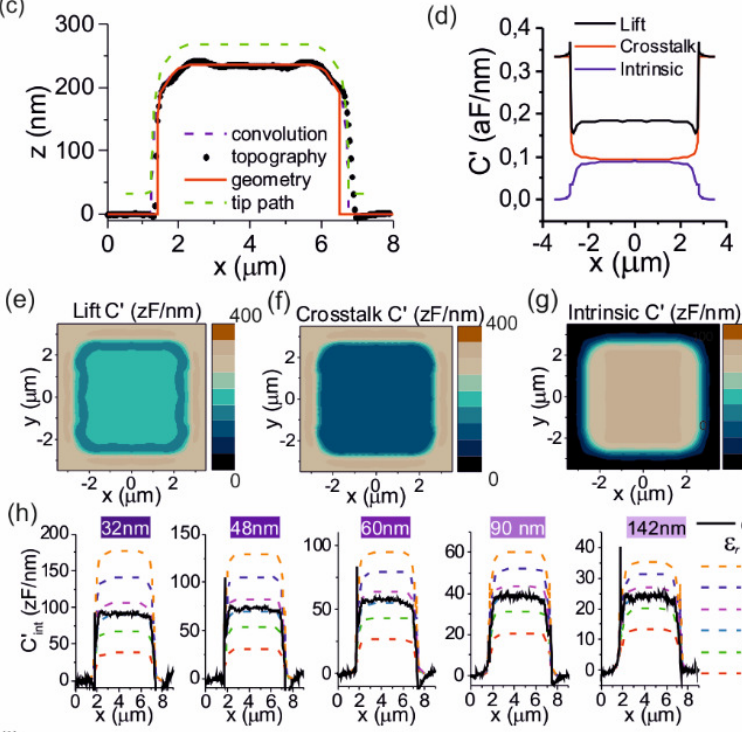

(g)

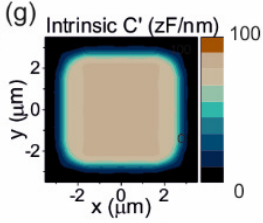

(i)
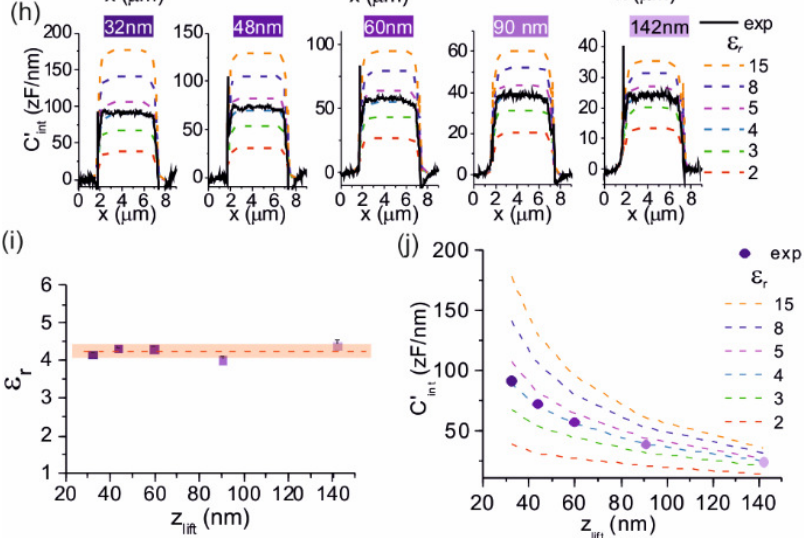

Figure 3. (a) Schematic representation (not to scale) of the system geometry modelled. Dimensions of the oxide pillar $w=5.2 \mu \mathrm{m}$ and $h=236 \mathrm{~nm}$. Tip geometry with calibrated radius $R=167 \mathrm{~nm}$ and half cone angle $\theta=15^{\circ}$, and nominal values $H=12.5 \mu \mathrm{m}, W=3 \mu \mathrm{m}, L=0 \mu \mathrm{m}$. (b) Green surface: ensemble of simulated lines representing the 3D tip movement over the oxide pillar (convoluted topography). The brown surface represents the actual oxide pillar geometry simulated. (c) Comparison of a measured topographic profile (symbols) with a tip convoluted profile (dashed purple) obtained for the pillar and tip geometries simulated (red line). Also shown the tip path followed during the simulations (green line) for a lift distance $z_{\text {lift }}=32 \mathrm{~nm}$. (d) Transversal cross-section profiles along the center of the numerically calculated images in (e)-(g), in absolute values. (e) Numerically calculated lift capacitance gradient image, (f) topographic crosstalk capacitance gradient image (obtained by setting $\varepsilon_{r}=1$ in the simulations), and (g) intrinsic capacitance gradient image obtained from the subtraction of (f) from (e). (h) Series of numerically calculated intrinsic capacitance gradient profiles for different dielectric constants of the pillar (dashed lines) and different lift distances (from left to right $32 \mathrm{~nm}, 48 \mathrm{~nm}, 60 \mathrm{~nm}, 90 \mathrm{~nm}$ and $142 \mathrm{~nm}$ ). Also shown the experimental intrinsic capacitance gradient profiles measured at each lift distance (continuous thick lines). For all distances the experimental profiles agree with $\varepsilon_{r} \sim 4$. The experimental profiles in the image are the average of 10 consecutive lines for better signal to noise ratio. (i) Extracted electric permittivity values resulting from contrast values obtained from the intrinsic capacitance gradient images in figure $2 \mathrm{e}$, as a function of lift distance. The average value obtained is $\varepsilon_{r} \sim 4.2 \pm 0.2$ (j) (Dashed lines) Numerically calculated intrinsic capacitance gradient contrast curves on the center of the pillar as a function of lift distance. (Symbols) Experimental contrast values obtained from the intrinsic capacitance gradient images. A least square fitting - 5 of the data gives $\varepsilon_{r}=4.1 \pm 0.2$. 
calculated data (associated to non-local cantilever effects), and fitted together with the tip radius and cone angle. An example of a fitted curve is shown in figure $2 \mathrm{~g}$, where the black continuous line represents the theoretically calculated curve that best fits the experimentally measured curve (red line). In this case, we obtain a tip radius $R=166 \pm 2 \mathrm{~nm}$ and half cone angle $\theta=15.0 \pm 0.5^{\circ}$, with a constant offset $k_{\text {stray }}=0.128 \pm 0.002$ $\mathrm{aF} / \mathrm{nm}$.

The pillar oxide is assumed to have a physical shape and dimensions consistent with the measured topographic images, after proper subtraction of tip dilation effects. The tip dilation effects have been calculated for the tip geometrical dimensions (determined by the tip calibration process described above). Based on this analysis, the pillar has a thickness $h=236 \mathrm{~nm}$ and a width $w=5.2 \mu \mathrm{m}$, with round edges at the top and lateral sides (brown pillar in figure $3 \mathrm{~b}$ ). The assumed pillar geometry provides an excellent agreement with the measured topographic image: see figure $3 \mathrm{c}$ where we compare the calculated tip dilated profile of the assumed geometry (blue dashed line) with the measured topographic profile (symbols). For comparison, we also show the physical geometry of the pillar (red line) and the tip path when in lift imaging (green line). Finally, a uniform relative dielectric constant, $\varepsilon_{r}$, is assumed for the oxide based on the analysis of the intrinsic capacitance gradient images.

The capacitance gradient between the tip and sample is calculated by solving Poisson's equation with the finite element numerical software Comsol Multiphysics 5.2 (AC/DC electrostatic module). Poisson's equation solution results in the distribution of the static electric potential around the tip and in the sample, from which we derive the Maxwell stress tensor on the tip surface, and, by integration of it on the surface of the tip, we obtain the electrostatic force (see further details elsewhere [35]). The mesh was set to at least 200000 elements. An accurate process of optimization, validation and numerical noise reduction of the $3 \mathrm{D}$ simulations has been undertaken, in order to meet the experimental requirements and to enable the handling of 3D structures whose physical dimensions vary in more than three orders of magnitude.

Lift mode capacitance gradient images $C^{\prime}\left(\vec{x}, z_{\text {lift }}\right)$ have been computed with the model geometry described above. To this end, software routines written in Matlab (Mathworks Inc.) have been used to move the tip in the simulations with respect to the pillar following the (convoluted) topographic profile at the set lift distance, i.e, $z(\vec{x})=h(\vec{x})+z_{\text {lift }}$ (green surface in figure 3b). Images of $45 \times 51$ points have been calculated. Images for the five experimental lift distances $z_{\text {lift }}=32 \mathrm{~nm}, 48 \mathrm{~nm}, 59 \mathrm{~nm}, 90$ $\mathrm{nm}$ and $142 \mathrm{~nm}$ have been calculated. For each lift distance a range of dielectric constant values has been considered $\left(\varepsilon_{r}=1-15\right)$. An example of a calculated capacitance gradient lift image for $z_{\text {lift }}=32 \mathrm{~nm}$ and $\varepsilon_{r}=4$ is shown in figure $3 \mathrm{e}$, together with its cross-section profile in figure $3 d$ (black line). Absolute values are shown. As it can be seen, the image shows the same trends as the experimental image, although the absolute values do not match the experimental ones, since the model does not include the non-local cantilever stray effects. By simply setting $\varepsilon_{r}=1$ in the simulations we can calculate the topographic crosstalk images. An example for $z_{\text {lift }}=32 \mathrm{~nm}$ is shown in figure $3 \mathrm{f}$, together with a cross-sectional profile in figure $3 \mathrm{c}$ (red line). Again, the qualitative trend is similar to the images derived from the experiments (see figure 2), but a quantitative match is not obtained because of the reason mentioned above. Finally, by subtracting the topographic crosstalk capacitance gradient images from the lift images we obtain the numerically calculated intrinsic capacitance gradient images. An example of a calculated intrinsic capacitance gradient image for $z_{\text {lift }}=$ $32 \mathrm{~nm}$ and $\varepsilon_{r}=4$, is shown in figure $3 \mathrm{~g}$, together with a cross-sectional profile in figure $3 \mathrm{c}$ (blue line). The image also shows a similar behavior to the experimental intrinsic capacitance gradient images (figure 2). In this case the agreement with the experimental results is quantitative, since the intrinsic capacitance gradient image is not affected by non-local stray effects related to the cantilever (which as we have mentioned are not included in the theoretical model). Comparison of the experimental profiles obtained from the intrinsic images (black solid lines, same profiles as blue lines in figure $2 \mathrm{f}$ ) at the five lift distances considered with the theoretical ones (dashed lines) for different dielectric constants of the oxide are shown in figure $3 \mathrm{~h}$. For all lift distances the profiles approach the curves corresponding to $\varepsilon_{\mathrm{r}} \sim 4$, independently of the lift distance. The values obtained for the dielectric constants for the different distances is shown in figure $3 \mathrm{i}$. These values were derived by calculating the intrinsic capacitance contrast between the center of the pillar (average of 300 pixels) and the substrate (average of 800 pixels) from the images in $2 \mathrm{e}$, and fitting these values with the corresponding theoretical contrast as a function of the dielectric constant, at each $z_{\text {lift }}$. The overall average value is $\varepsilon_{r}=4.2 \pm 0.2$. A similar value is obtained if one performs a least square fitting of the intrinsic capacitance gradient contrast in the center of the pillar as a function of the lift distance, with the correspondingly theoretically calculated curves for different dielectric constants (see figure $3 \mathrm{j}$ ). In this case a value $\varepsilon_{r}=4.1 \pm 0.2$ is obtained. These values of the electric permittivity are in full agreement with the value usually reported for silicon 
dioxide, thus confirming the quantitative accuracy of the proposed procedure and, hence, validating it. We have further validated the method with the case of polystyrene nanoparticles of diameter $\sim 300 \mathrm{~nm}$, similar to the height of the silicon dioxide pillars. Also in this case, we obtain an excellent agreement between the extracted dielectric permittivity and the nominal value usually quoted for polystyrene (details are provided in the Supplementary Information).

\section{Application to single bacterial cell}

We show the usefulness of the proposed approach for the case of a sample showing a relatively complex nonplanar surface geometry, namely, a single bacterial cell under dry conditions. In particular, we consider the case of bacterial cells of the Bacillus Cereus CECT495 type, endospore forming bacteria, in an early sporulation state [36]. The bacterial cells have been grown following the procedure detailed in Ref. [37]. Briefly, an individual colony of B. cereus CECT 495 was grown for $24 \mathrm{~h}$ at $30^{\circ} \mathrm{C}$ at $250 \mathrm{rpm}$ in Trypticase soy broth. For early stage sporulation, $0.1 \mathrm{ml}$ of the culture was added into $25 \mathrm{ml}$ of G Medium [38] and incubated at $30^{\circ} \mathrm{C}$ and $250 \mathrm{rpm}$ for 8h. Sample was rinsed in milli-Q water at a low centrifugation $\left(4 \mathrm{~min}, 4^{\circ} \mathrm{C}, 4000 \mathrm{rpm}\right)$ and deposited on a gold substrate (Arrandee), previously cleaned following sequential sonication washing with acetone, isopropanol and water. Samples were allowed to dry in a cabin flow and imaged under nitrogen ambient flow ( $\sim \%$ Relative Humidity) .

Figure 4a shows a 3D representation of the topographic image of the bacterial cell, together with longitudinal and transversal cross-section profiles. As it can be seen the bacterial cell surface topography is highly non-planar and, then, suitable for the analysis proposed here.

Besides the evident surface rough variations, the bacterial geometry approximately adapts to an ellipsoidal cap geometry. This fact is illustrated in the profiles shown in figure $4 \mathrm{a}$ where we compare the tip convoluted profiles (blue lines, practically indistinguishable from the red lines) obtained from the convolution of the tip (with its calibrated geometry) and a cap ellipsoid of width $w=$ $1499 \mathrm{~nm}$, length $l=4496 \mathrm{~nm}$ and height $h=250 \mathrm{~nm}$ (red lines in the profile panels in figure $4 \mathrm{a}$, see Appendix A for more details). We note that this geometry is very close to the hemiellipsoid geometry used in Ref. [30], but adapts slightly better to the geometry of the bacterial cell investigated here. The tip dimensions have been obtained from the calibration curve measured on the substrate shown in figure $4 \mathrm{~b}$, giving $R=115 \pm 1 \mathrm{~nm}, \theta=30.0 \pm 0.3^{\circ}$ and $k_{\text {stray }}=0.108 \pm 0.002 \mathrm{aF} / \mathrm{nm}$.

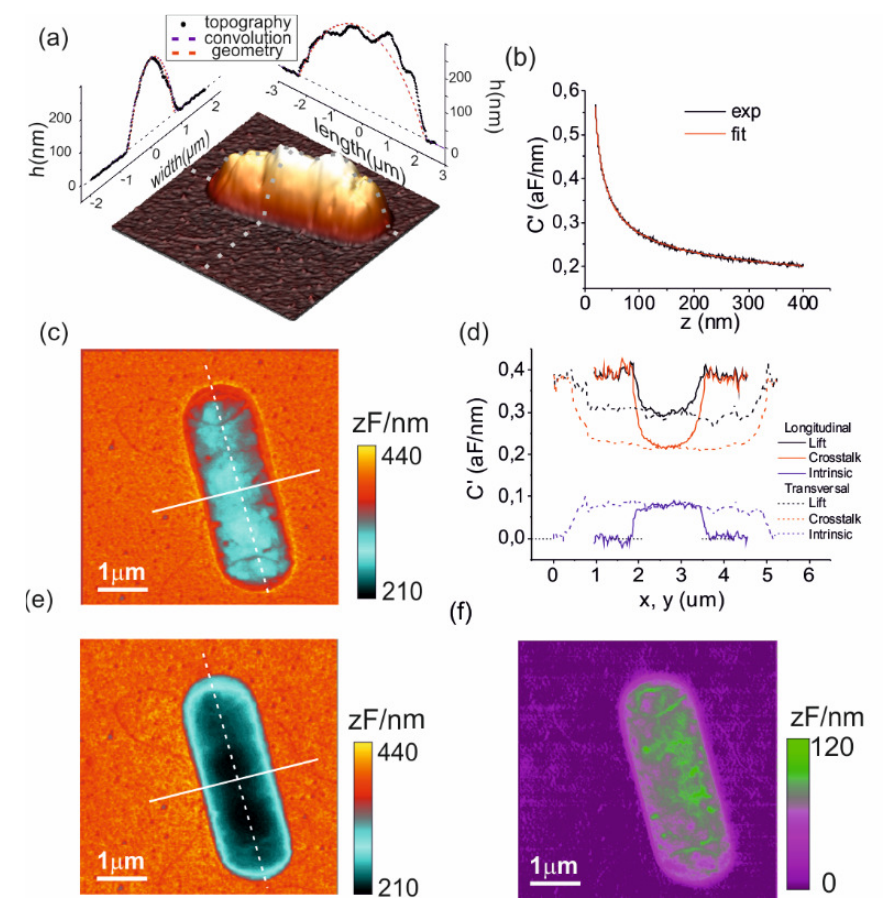

Figure 4. (a) 3D reconstruction of a topographic image of a Bacillus Cereus bacterial cell, together with transversal and longitudinal cross-section profiles. Also shown in the profiles panels the convoluted profiles (dashed lines) obtained from the convolution of the tip with an ellipsoidal cap of width $1448 \mathrm{~nm}$, length $4456 \mathrm{~nm}$ and height $250 \mathrm{~nm}$. (b) Capacitance gradient approach curve on the metallic substrate (symbols) and corresponding theoretical least square fitting curve (black line). The fits gives $R=115 \pm 1 \mathrm{~nm}$, $\theta=30.0 \pm 0.3^{\circ}$ and $k_{\text {stray }}=0.108+0.002 \mathrm{aF} / \mathrm{nm}$. (c) Lift-mode capacitance gradient EFM image obtained at a lift distance of $38 \mathrm{~nm}$. (d) Cross-sectional profiles along the lines in (b) (black lines), in (e) (red lines) and in (f) (blue lines). (e) Topographic crosstalk capacitance gradient image reconstructed from the topographic image in (a) and the approach curve in (b). (f) Intrinsic capacitance gradient image obtained from the subtraction of (e) from (c). Note the change in scale range with respect to (c) and (e).

Capacitance gradient lift-mode EFM images have been measured at five different lift distances, $z_{\text {lift }}=38 \mathrm{~nm}, 49$ $\mathrm{nm}, 60 \mathrm{~nm}, 79 \mathrm{~nm}$ and $127 \mathrm{~nm}$. Figure $4 \mathrm{c}$ shows one example of a capacitance gradient lift-mode EFM image (in absolute values) acquired at a lift distance $z_{\text {lift }}=38 \mathrm{~nm}$. Similarly to the case of the oxide pillar, the absolute capacitance gradient decreases when moving from the substrate to the top of the bacterial cell (see the transversal and longitudinal cross sectional profiles in figure $4 \mathrm{~d}$, continuous and dotted black lines, respectively). In the present case we remark the presence of features in the electrical image on the bacterial surface. Whether such features correspond to bacterial inhomogeneities or to topographic crosstalk effects can be investigated by analyzing the intrinsic capacitance gradient image. To obtain it, we first constructed the topographic crosstalk capacitance gradient image with the help of the topographic image shown in figure $4 \mathrm{a}$ and the capacitance 
gradient approach curve measured on the bare substrate (shown in figure $4 \mathrm{~b}$, black line). The result is shown in figure $4 \mathrm{e}$, together with transversal and longitudinal cross-section profiles shown in figure $4 \mathrm{~d}$ (continuous and dotted red lines, respectively). As it can be seen, the topographic crosstalk image presents also electrical features on the bacterial cell surface, showing that most of the features observed in the lift capacitance gradient image come from topographic crosstalk effects. By subtracting the topographic crosstalk from the lift image, we obtain the intrinsic capacitance gradient image, shown in figure 4f. The intrinsic capacitance gradient image shows a fairly uniform (compared to the previous images) and positive contrast indicating the lack of relevant electrical inhomogeneities. In fact, the intrinsic capacitance gradient transversal and longitudinal cross sectional profiles are fairly flat (figure $4 \mathrm{~d}$, continuous and dotted blue lines, respectively), indicating the lack of contributions of both large and small topographic variations, as compared to the topographic profiles (figure 4a) or lift capacitance gradient profiles (figure $4 d$, black lines).

We quantified the intrinsic capacitance gradient images by means of 3D finite element numerical simulations by using a system geometry similar to the one used for the oxide pillars, but substituting the oxide pillar by a bacteria with an ellipsoidal cap shape (see figure 5a). The dimensions of the bacterial cell are those obtained from the tip deconvolution analysis described above $(w=1499$ nm, $l=4496$ and $h=250$ ), and those of the tip, from the tip calibration procedure, also described before, $(R=$ $115 \pm 1 \mathrm{~nm}$ and $\left.\theta=30.0 \pm 0.3^{\circ}\right)$. Lift capacitance gradient images of $91 \times 35$ points following the bacterial cell lifted convoluted topography (green lines in figure $5 b$ ) have been calculated for the five experimental lift distances $z_{\text {lift }}$ $=38 \mathrm{~nm}, 49 \mathrm{~nm}, 60 \mathrm{~nm}, 79 \mathrm{~nm}$ and $127 \mathrm{~nm}$ and different dielectric constants in the range $\varepsilon_{r}=2-15$. An example for the lift distance $z_{\text {lift }}=38 \mathrm{~nm}$ and $\varepsilon_{r}=6$ is shown in figure 5c. By setting $\varepsilon_{r}=1$ in the calculations we obtained the corresponding topographic crosstalk capacitance gradient images (see the one corresponding to $z_{\text {lift }}=38 \mathrm{~nm}$ in figure 5d). Subtracting the crosstalk from the lift capacitance gradient images, we obtain the intrinsic capacitance gradient images (see the one for $z_{\text {lift }}=38 \mathrm{~nm}$ and $\varepsilon_{r}=6$ in figure $5 \mathrm{e}$ ). The respective transversal cross-section profiles are shown in figure $5 \mathrm{f}$. Note the fairly flat profile of the intrinsic capacitance gradient image. The intrinsic capacitance gradient images can be compared directly with the experimental ones. In figure $5 \mathrm{~g}$ we show the calculated intrinsic capacitance gradient transversal profiles (dashed lines) at the different lift distances, for different dielectric constants of the bacterial cell, and (a)
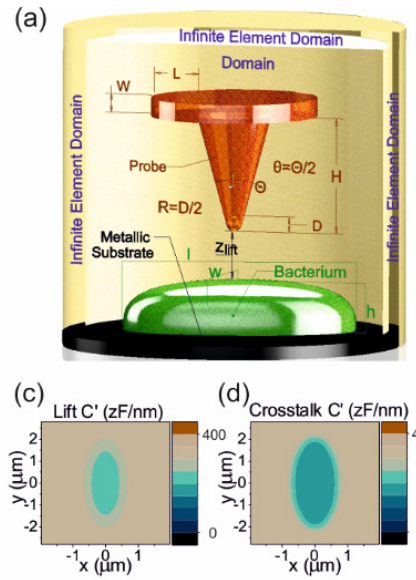

(d) Crosstalk C' (zF/nm)

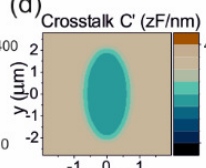

(b)

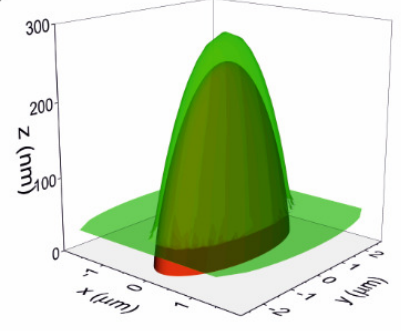

(e) Intrinsic

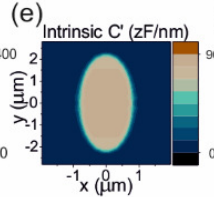

(f)

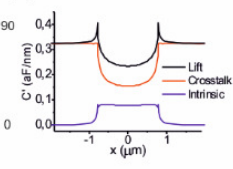

(g)

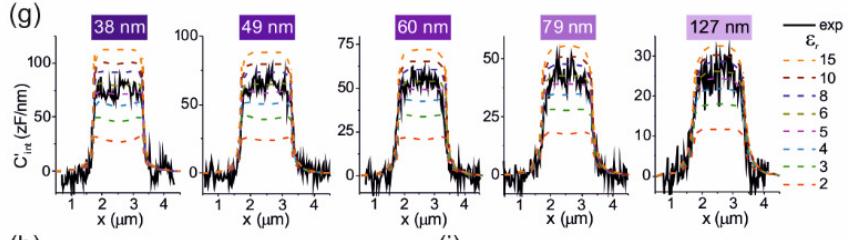

(h)

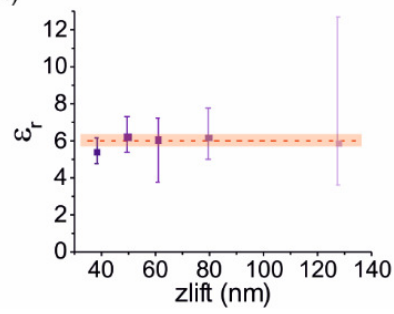

\section{(i)}

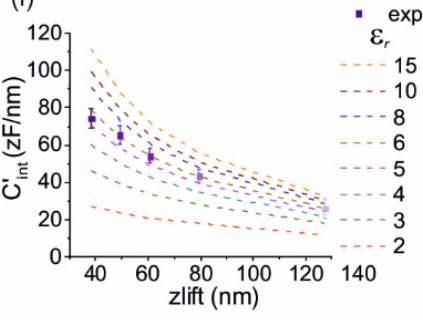

Figure 5. (a) Schematic representation (not to scale) of the system geometry modelled for the bacterium. Dimensions of the bacterium $w=1.499 \mu \mathrm{m}, l=$ $4.496 \mu \mathrm{m}$ and $h=250 \mathrm{~nm}$. Tip geometry with calibrated radius $R=115 \mathrm{~nm}$ and half cone angle $\theta=30^{\circ}$, and nominal values $H=12.5 \mu \mathrm{m}, W=3 \mu \mathrm{m}, L=$ $0 \mu \mathrm{m}$. (b) Green surface: ensemble of simulated lines representing the 3D tip movement over the bacterium (convoluted topography). The red surface represents the actual bacterial cell geometry simulated. (c) Numerically calculated lift capacitance gradient image for $z_{\text {lif }} t=38 \mathrm{~nm}$ and $\varepsilon_{r}=6$, and corresponding topographic crosstalk capacitance gradient image (d), obtained by setting $\varepsilon_{r}=1$ in the simulations, and intrinsic capacitance gradient image (e), obtained from the subtraction of (d) from (c). Images are of 91x35 pixels. (f) Transversal crosssection profiles along the center of the images in (c), (d) and (e). Absolute values are shown. (g) Comparison of the measured intrinsic capacitance gradient profiles at five different lift distances (continuous lines) with numerically calculated profiles for different dielectric constant values (dashed lines). (h) Extracted dielectric constant at each lift distance after matching the theoretical values to the experimental ones obtained on the centre of the bacterial cell. The average value is $\varepsilon_{r}=6.0 \pm 0.3$. (i) Intrinsic capacitance gradient contrast in the centre of the bacterial cells as a function of lift distance (symbols), compared with numerically calculated curves for different dielectric constants of the bacterial cell (dashed lines). The best agreement is found for $\varepsilon_{r}=5.8 \pm 0.4$.

compare them with the corresponding experimental intrinsic capacitance gradient profiles (black solid lines). From this comparison, we obtain $\varepsilon_{r} \sim 5-6$. A more precise estimation for each lift distance is obtained by matching the average value obtained on the center of the bacterium (60 pixels) with the corresponding theoretical values as a function of the dielectric constant of the bacterial cells. 
We obtain in all cases values in the range $\varepsilon_{r} \sim 5.5-6.5$ (see figure $5 \mathrm{~h}$ ) with an average value $\varepsilon_{r}=6.0 \pm 0.3$. A similar value $\left(\varepsilon_{r}=5.8 \pm 0.3\right)$ is obtained from a least square fitting of the intrinsic contrast values as a function of lift distances with theoretically calculated intrinsic contrast curves on the center of the bacterial cell for different dielectric constants (figure 4i).

\section{Discussion}

We have presented a method to quantify capacitance gradient EFM images obtained in lift mode on highly nonplanar samples and to obtain the local dielectric constant of this type of samples. To this end we have introduced the concept of intrinsic capacitance gradient image, which is obtained from the subtraction of the topographic crosstalk capacitance gradient image from the lift capacitance gradient image. The use of the intrinsic capacitance gradient images offers several advantages from the point of view of obtaining information on the local dielectric permittivity properties of non-planar samples and to extract quantitative values for their electric permittivity. First, it enables to directly visualize the homogeneity (or heterogeneity) of the sample's dielectric properties not masked by topographic artefacts with the highest signal to noise ratio (i.e. with the tip as close as possible to the sample in all points of the image). This property is important as it allows inferring local dielectric permittivity properties of the sample not perturbed with topographic properties. Second, it enables to set up theoretical sample models that are well adapted to the actual dielectric permittivity properties of the sample (for instance, in the cases analysed here, both samples showed a relatively uniform dielectric response in the intrinsic images, what justified the use of uniform dielectric theoretical models). And third, it allows one to use simplified geometrical probe models, since the contribution of microscopic parts not related to the dielectric permittivity properties of the sample (e.g. cantilever) are subtracted from this type of images and they do not need to be included into the calculations.

The proposed approach offers clear advantages when applied to highly non-planar samples with respect to other approaches reported in the literature. For instance, we and other authors [3,26,31] have suggested the use of constant height imaging mode, in which the tip substrate distance is kept constant during image acquisition. This imaging mode produces electric images free from topographic crosstalk contributions and, hence, are also directly amenable to a quantitative interpretation in terms of the local polarizability of the sample [3,26]. However, for samples showing large topographic variations (in the hundreds nanometer or micrometric ranges) the constant height imaging mode only provides accurate electric information on the highest parts of the sample, since the remaining parts lie at a too large distance from the tip during the image acquisition. This fact prevents accessing the local dielectric properties in all parts of the sample with high accuracy. The use of lift mode imaging ensures the maximum sensitivity in all positions of the sample, and our analysis ensures the results are free from topographic crosstalk artefacts. We would like to highlight, however, that for planar samples or low dimensional non-planar samples (like nanoparticles, nanotubes, etc,) the use of constant height imaging mode can be preferred since the accuracy required (very often in the sub-1zF/nm) $[26,27,29]$ cannot be offered by the reconstruction procedure presented here.

The proposed method has been validated with measurements on $\mathrm{SiO}_{2}$ pillars, providing a value in excellent agreement with values quoted for high quality $\mathrm{SiO}_{2}$ oxides $\left(\varepsilon_{r} \sim 4\right)$. For the case of the $B$. Cereus bacterial cell, the value obtained $\left(\varepsilon_{r} \sim 6\right)$ is slightly larger than the values reported earlier for other types of bacteria cells ( $S$. Thyphimurium, E. Coli, L. Sakey and L. innocua) in the same dry conditions $\left(\varepsilon_{r} \sim 3-5\right)$ [30]. A possible explanation for the higher effective polarization of $B$. Cereus could be related to the initial sporulation state of the bacterial cell, in which the cell wall tends to thin and the DNA content (which shows a relatively large polarizability $\left(\varepsilon_{r} \sim 8\right)$ [27]) tends to increase. Indeed, in this type of bacterial cell, after the triggering of spore formation due to harsh conditions, the first step is DNA replication [36]. After this step has ended, the replicated DNA goes to one end of the bacterial cell and it is encapsulated by spore layers until it becomes a mature endospore. At the early stage of spore formation we use (8h) it has been reported that the spore is not present yet, since it only appears at around $12 \mathrm{~h}$ in sporulation medium [37]. Instead, a process of reduction/loss of the thick cell wall characteristic of gram positive bacterial cells is occurring, which manifest in a significant reduction in bacteria height from the usual $\sim 500 \mathrm{~nm}$ in dry conditions to the $\sim 265 \mathrm{~nm}$ observed by us.

Finally, we remark that for the geometry and dimensions of the bacterial cells studied here, the use of 3D numerical simulations is unavoidable in order to obtain accurate values of the dielectric constant (beyond their need to simulate full images and profiles). In our previous work, we used equivalent $2 \mathrm{D}$ axisymmetric models preserving the bacterial cell volume and height [30] (i.e. representing hemiellipsoids by equivalent hemispheroids). We will show below that this approximation is valid only when the aspect ratio of the bacterial cell is small (e.g. length:width<2:1), like those of the bacterial cells 
analyzed in Ref. [30]. In the case of the B. Cereus cells analyzed here the length is nearly double (close to $4 \mu \mathrm{m}$ ) while the height and width are similar to the other bacterial types, thus giving an aspect ratio
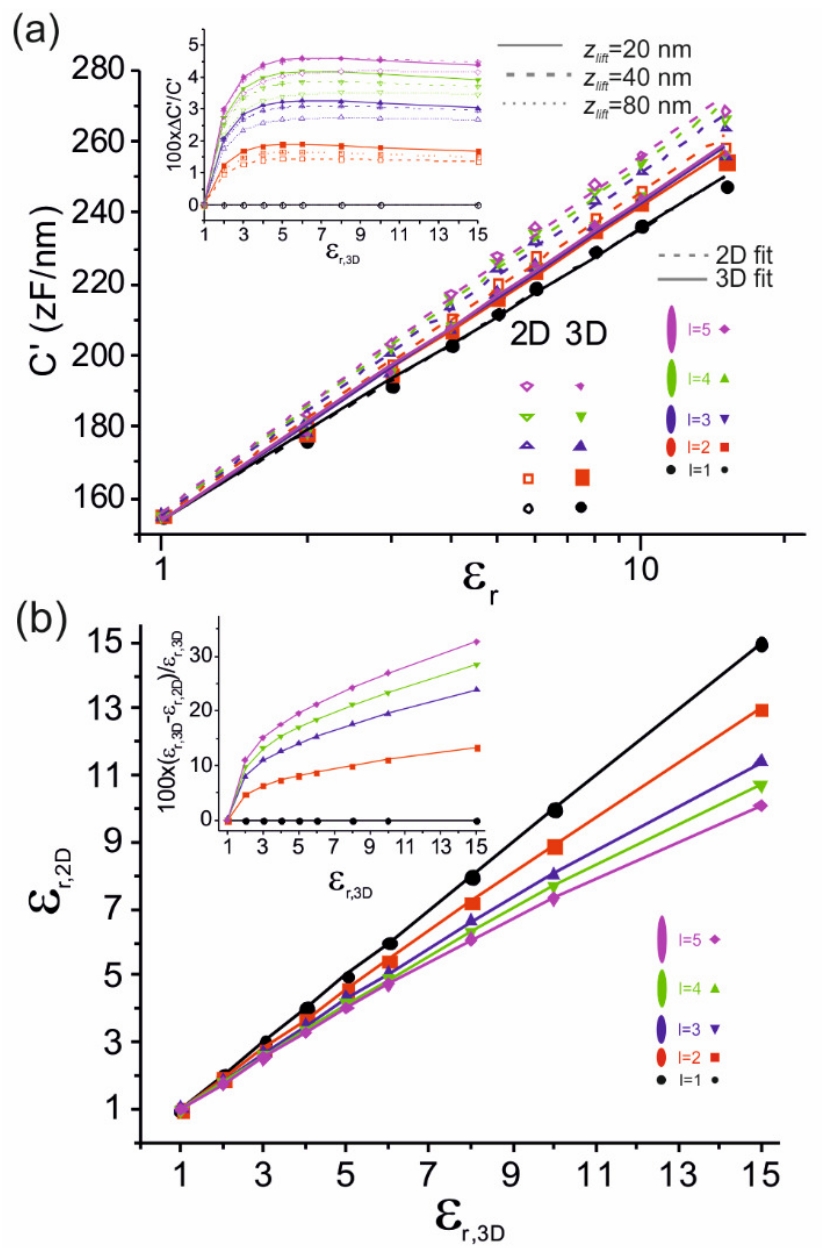

Figure 6. (a) Linear-log representation of calculated absolute capacitance gradient values on the center of the bacterial cell as a function of the dielectric constant of the cell for different hemiellipsoidal geometries 3D (filled symbols) and their equivalent hemispheroid geometries with same volume and height 2D (empty symbols). The continuous and dashed lines represent linear$\log$ fits of the calculated data. Inset: Relative error between the capacitance gradients calculated with the $3 \mathrm{D}$ and the $2 \mathrm{D}$ models as a function of the dielectric constant, for different bacterial lengths and three different lift distances, $z_{\text {lift }}=20 \mathrm{~nm}, 40 \mathrm{~nm}$ and $80 \mathrm{~nm}$. (b) Dielectric constant extracted by using the 2D model using as input data the values calculated with the 3D model as a function of the dielectric constant, for the five bacterial cells considered. Inset: Relative error on the extracted dielectric constant of the 2D model as a function of the dielectric constant for the five bacterial lengths considered. Tip geometry: $R=150 \mathrm{~nm}, \theta=15^{\circ}, H=12.5, W=3 \mu \mathrm{m}, L=0 \mu \mathrm{m}$. Bacterial cell geometry: width $w=1 \mu \mathrm{m}$, height $h=250 \mathrm{~nm}$ and lengths $l=1 \mu \mathrm{m}, 2 \mu \mathrm{m}, 3 \mu \mathrm{m}$, $4 \mu \mathrm{m}$ and $5 \mu \mathrm{m}$.

length:width>2:1. For these geometries, the 2D axisymmetric approximation are not accurate enough to enable extracting reliable dielectric constant values.

We show it explicitly in figure 6, where we compare calculated capacitance gradient values on the center of the bacterial cell at a lift distance $z_{l i f t}=40 \mathrm{~nm}$ as a function of the dielectric constant of the bacterial cell, for different 3D hemiellipsoidal geometries (solid symbols) and their corresponding equivalent 2D hemispheroid geometries (empty symbols). The 3D bacterial cells have the same height $h=250 \mathrm{~nm}$ and width $w=1 \mu \mathrm{m}$, and different length $(l=1,2,3,4$ and $5 \mu \mathrm{m})$. For the 2D geometries the radius of the equivalent hemispheroids are $R_{e q}=500 \mathrm{~nm}$, $707 \mathrm{~nm}, 866 \mathrm{~nm}, 1000 \mathrm{~nm}$ and $1118 \mathrm{~nm}$, respectively. The equivalent $2 \mathrm{D}$ model tends to slightly overestimate the capacitance gradient values as compared to the 3D model, providing values between $1 \%$ and $5 \%$ larger (see inset figure $6 \mathrm{a}$ where we plot the relative error between both models as a function of the dielectric constant for the different bacterial lengths, and for different lift distances $z_{\text {lift }}=20 \mathrm{~nm}, 40 \mathrm{~nm}$ and $80 \mathrm{~nm}$ ).

Even if the relative error in the calculated capacitance gradients is relatively small, the extracted dielectric constants show much larger errors between the two models. We show it in figures $6 \mathrm{~b}$, where we plot the dielectric constant that would be obtained by using the 2D model to quantify the simulation data obtained with the 3D model (see also the inset where the relative error is calculated). We observe that only for small aspect ratios $<2: 1$ for which the calculated capacitance gradients errors between the $2 \mathrm{D}$ and $3 \mathrm{D}$ models are below $\sim 2 \%$, the relative error in the extracted $2 \mathrm{D}$ dielectric constant is below $\sim 10 \%$ (for $\varepsilon_{r}<10$ ), which is an acceptable uncertainty. However, for aspect ratios larger than 2:1 the relative error in the calculated capacitance gradients is above a $\sim 2 \%$ and the relative error in the extracted dielectric constant can grow up to $\sim 28 \%$ for the more eccentric geometries $(l=5 \mu \mathrm{m})$. Such large errors can be avoided by using 3D models to quantify the measurements for these type of bacterial cells, as we did here. The reason why the extracted dielectric constants are so sensitive to the actual intrinsic capacitance gradient values is that they depend logarithmically on the dielectric constant (dashed and continuous lines in figure 6a). This fact makes that the relative error in the extracted dielectric constant depend exponentially on the relative error in the calculated intrinsic capacitance gradients (see Appendix B),

$\frac{\Delta \varepsilon_{r}}{\varepsilon_{r}} \approx 1-\exp \left[-\frac{\Delta C_{\text {int }}^{\prime}}{C_{\text {int }}^{\prime}} \ln \left(\varepsilon_{r}\right)\right]$

\section{Conclusions}

We have presented a quantitative analysis of lift mode electrostatic force microscopy images for nanoscale dielectric characterization. We have shown that for highly non-planar samples a significant contribution to the images comes from the topographic crosstalk associated 
to the variation of the tip-substrate distance while the tip tracks the sample topography. A method to subtract this contribution from the images has been presented. The resulting image has been shown to reflect more precisely the local dielectric properties of the sample. Application of this procedure to experimental results obtained on silicon dioxide pillars and on single bacterial cells fully confirm the need of the proposed method for quantitative analysis of the dielectric properties of highly non-planar samples. Present results are expected to be especially useful in samples showing large topographic variations, such as dielectric samples with high steps or single cells, where the topographic crosstalk contribution can mask the intrinsic dielectric response of the sample.

\section{Acknowledgments}

This work has been partially supported by the Nanomicrowave project funded from the European Union Seventh Framework Programme (FP7/People-2012-ITN) under grant agreement $n^{\circ} 317116$.EU, and also by the Spanish project TEC2013-48344-C2. (GG) acknowledges support from an ICREA Academia grant from the Generalitat de Catalunya. We acknowledge R. Millán-Solsona for technical support, and Prof. A. Juárez, from University of Barcelona, for assistance in bacterial sample preparation.

Appendix A: Tip dilation analysis for a cap ellipsoid For a cap ellipsoid corresponding to an ellipsoid of semiaxes $a, b$ and $c$, buried under the surface a depth $d$ (the hemiellipsoid correspond to $d=0$ ), the coordinates of a transversal cross-section profile $z(x)$ representing the convolution between the tip apex of radius $R$ and the bacterial cell are given by:

$$
z(x)=c \cdot \sin \alpha(x)-\frac{a}{2} \tan \alpha(x) \cdot \sqrt{\frac{R^{2}}{1+\left(\frac{a}{c} \tan \alpha(x)\right)^{2}}}-R-d
$$

where only $z(x)>0$ are considered and where the angle $\alpha(x)$ is found for any $x \in[-(a+R), a+R]$ from the roots of the equation of the centre $x$ of the apex tangent to the cap ellipse in the point of abscissa $a \cos \alpha(x)$, i.e.,

$$
x=a \cos \alpha(x)-\sqrt{\frac{R^{2}}{1+\left(\frac{a}{c} \tan \alpha(x)\right)^{2}}}
$$

(Similarly for the $z(y)$ profile, substituting $a$ by $b$ ).

The deconvoluted dimensions of the cap ellipsoid are obtained by adjusting equation (A1) to the measured topographic profile, by varying the parameters $b, c$ and $d$ (for the transversal profile) and $a, c$ and $d$ for the longitudinal profile, leaving the difference $c-d$ fixed to the measured height. In particular, for the bacterial cell shown in figure 4 a we obtained an ellipsoid of semiaxes $a=1000 \mathrm{~nm}, b=3000 \mathrm{~nm}$ and $c=740 \mathrm{~nm}$, "buried" a distance $d=490 \mathrm{~nm}$.

The method holds true only if the contact point between tip and cap ellipsoid belongs always to the surface of the tip apex, and not to the cone surface. For a cone angle $\theta$, this condition is mathematically verified if,

$$
R \cdot(1-\sin \theta) \geq \frac{1}{\sqrt{\frac{a^{2}}{c^{4}} \cot ^{2} \theta+\frac{1}{c^{2}}}}-d
$$

\section{Appendix B: Relative error in the extracted dielectric constants between $2 \mathrm{D}$ and $3 \mathrm{D}$ models}

According to the calculations shown in figure $6 \mathrm{a}$, the intrinsic capacitance gradient in the center of the bacterial cell for both the 3D and the 2D models follows an approximate logarithmic dependence on the dielectric constant for $\mathcal{E}_{r}<10$ (dashed and continuous lines in figure 6a) i.e.

$$
\begin{aligned}
& C_{\mathrm{in}, 3 D}^{\prime}\left(z, \varepsilon_{r}\right)=C_{3 D}\left(z, \varepsilon_{r}\right)-C_{3 D}\left(z, \mathcal{E}_{r}=1\right) \approx b_{3 D} \ln \left(\varepsilon_{r}\right) \\
& C_{\mathrm{int}, 2 D}\left(z, \mathcal{E}_{r}\right)=C_{2 D}\left(z, \mathcal{E}_{r}\right)-C_{2 D}\left(z, \mathcal{E}_{r}=1\right) \approx b_{2 D} \ln \left(\varepsilon_{r}\right)
\end{aligned}
$$

Here, $b_{2 D}$ and $b_{3 D}$ are parameters dependent on the geometry and tip sample distance but independent from the dielectric constant. The relative error in the calculated intrinsic capacitance gradients between the $3 \mathrm{D}$ and $2 \mathrm{D}$ models is then given by

$$
\frac{\Delta C_{\mathrm{int}}^{\prime}}{C_{\mathrm{in}, 2 D}}=\frac{C_{\mathrm{int}, 2 D}\left(z, \varepsilon_{r}\right)-C_{\mathrm{int}, 3 D}\left(z, \varepsilon_{r}\right)}{C_{\mathrm{int}, 2 D}^{\prime}\left(z, \varepsilon_{r}\right)} \approx \frac{b_{2 D}-b_{3 D}}{b_{2 D}}
$$

which is approximately independent from the dielectric constant for $\mathcal{E}_{r}<10$ where the $\log$-linear fit is correct (see figure 6a). For a given intrinsic capacitance gradient value, $C^{\prime}{ }^{\prime}$ intexp the extracted dielectric constants from the two models would be

$$
\varepsilon_{r, 2 D}=e^{\frac{C_{\text {intexp }}^{\prime}}{b_{2 D}}} ; \quad \varepsilon_{r, 3 D}=e^{\frac{C_{\text {interexp }}^{\prime}}{b_{3 D}}}
$$

The relative error of the $2 \mathrm{D}$ model with respect to the $3 \mathrm{D}$ one is then equal to

$$
\frac{\Delta \varepsilon_{r}}{\varepsilon_{r, 3 D}}=\frac{\varepsilon_{r, 3 D}-\varepsilon_{r, 2 D}}{\varepsilon_{r, 3 D}}=1-e^{-\frac{\Delta C_{\text {int }} \ln \left(\varepsilon_{r, 2 D}\right.}{C_{\text {in }}}}
$$

where use has been made of equations (B1)-(B3). This result is precisely equation (5) of the main text.

\section{References}

[1] Lee D T, Pelz J P and Bhushan B 2006 Scanning capacitance microscopy for thin film 
measurements Nanotechnology 17 1484-91

[2] Fumagalli L, Ferrari G, Sampietro M and Gomila G 2007 Dielectric-constant measurement of thin insulating films at low frequency by nanoscale capacitance microscopy Appl. Phys. Lett. 91243110

[3] Fumagalli L, Ferrari G, Sampietro M and Gomila G 2009 Quantitative nanoscale dielectric microscopy of single-layer supported biomembranes Nano Lett. 9 1604-8

[4] Krayev A V and Talroze R V 2004 Electric force microscopy of dielectric heterogeneous polymer blends Polymer (Guildf). 45 8195-200

[5] Crider P S, Majewski M R, Zhang J, Oukris H and Israeloff N E 2007 Local dielectric spectroscopy of polymer films Appl. Phys. Lett. 91013102

[6] Lu W, Wang D and Chen L 2007 Near-static dielectric polarization of individual carbon nanotubes. Nano Lett. 7 2729-33

[7] Riedel C, Arinero R, Tordjeman P, Lévêque G, Schwartz G a., Alegria a. and Colmenero J 2010 Nanodielectric mapping of a model polystyrenepoly(vinyl acetate) blend by electrostatic force microscopy Phys. Rev. E 81010801

[8] Fumagalli L, Gramse G, Esteban-Ferrer D, Edwards M a. and Gomila G 2010 Quantifying the dielectric constant of thick insulators using electrostatic force microscopy Appl. Phys. Lett. 96183107

[9] Kumar B, Bonvallet J C and Crittenden S R 2012 Dielectric constants by multifrequency noncontact atomic force microscopy. Nanotechnology 23025707

[10] Gomila G, Gramse G and Fumagalli L 2014 Finite-size effects and analytical modeling of electrostatic force microscopy applied to dielectric films. Nanotechnology 25255702

[11] Shao R, Kalinin S V. and Bonnell D A 2003 Local impedance imaging and spectroscopy of polycrystalline $\mathrm{ZnO}$ using contact atomic force microscopy Appl. Phys. Lett. 821869
[12] Pingree L S C and Hersam M C 2005 Bridgeenhanced nanoscale impedance microscopy Appl. Phys. Lett. 87233117

[13] Hu J, Xiao X-D and Salmeron M 1995 Scanning polarization force microscopy: A technique for imaging liquids and weakly adsorbed layers Appl. Phys. Lett. 67476

[14] Hu J, Xiao X D, Ogletree D F and Salmeron M 1995 Imaging the condensation and evaporation of molecularly thin films of water with nanometer resolution. Science $\mathbf{2 6 8} 267-9$

[15] Verdaguer A, Cardellach M and Fraxedas J 2008 Thin water films grown at ambient conditions on $\mathrm{BaF}(2)(111)$ studied by scanning polarization force microscopy. J. Chem. Phys. 129174705

[16] Benitez J J, de la Fuente O R, Díez-Pérez I, Sanz $F$ and Salmeron M 2005 Dielectric properties of self-assembled layers of octadecylamine on mica in dry and humid environments. J. Chem. Phys. 123104706

[17] Lai K, Ji M B, Leindecker N, Kelly M A and Shen Z X 2007 Atomic-force-microscopecompatible near-field scanning microwave microscope with separated excitation and sensing probes. Rev. Sci. Instrum. 78063702

[18] Lai K, Kundhikanjana W, Kelly M A and Shen Z-X 2011 Nanoscale microwave microscopy using shielded cantilever probes Appl. Nanosci. 1 $13-8$

[19] Cho Y 2011 Scanning nonlinear dielectric microscopy J. Mater. Res. 26 2007-16

[20] Krauss T D and Brus L E 1999 Charge, Polarizability, and Photoionization of Single Semiconductor Nanocrystals Phys. Rev. Lett. 83 4840-3

[21] Cherniavskaya O, Chen L, Weng V, Yuditsky L and Brus L E 2003 Quantitative Noncontact Electrostatic Force Imaging of Nanocrystal Polarizability J. Phys. Chem. B 107 1525-31

[22] Ben-Porat C H, Cherniavskaya O, Brus L, Cho K-S and Murray C B 2004 Electric Fields on Oxidized Silicon Surfaces: Static Polarization of 
PbSe Nanocrystals $\dagger$ J. Phys. Chem. A 1087814 9

[23] Bockrath M, Markovic N, Shepard A, Tinkham M, Gurevich L, Kouwenhoven L P, Wu M W and Sohn L L 2002 Scanned Conductance Microscopy of Carbon Nanotubes and $\lambda$-DNA Nano Lett. 2 187-90

[24] Lu W, Xiong Y, Hassanien A, Zhao W, Zheng $M$ and Chen L 2009 A Scanning Probe Microscopy Based Assay for Single-Walled Carbon Nanotube Metallicity Nano Lett. 9 166872

[25] Yang Y, Guo W, Wang X, Wang Z, Qi J and Zhang Y 2012 Size dependence of dielectric constant in a single pencil-like $\mathrm{ZnO}$ nanowire. Nano Lett. 12 1919-22

[26] Fumagalli L, Esteban-Ferrer D, Cuervo A, Carrascosa J L and Gomila G 2012 Label-free identification of single dielectric nanoparticles and viruses with ultraweak polarization forces Nat Mater 11 808-16

[27] Cuervo A, Dans P D, Carrascosa J L, Orozco M, Gomila G and Fumagalli L 2014 Direct measurement of the dielectric polarization properties of DNA. Proc. Natl. Acad. Sci. U. S. A. $111 \mathrm{E} 3624-30$

[28] Biagi M C, Fabregas R, Gramse G, Van Der Hofstadt M, Juárez A, Kienberger F, Fumagalli L and Gomila G 2016 Nanoscale Electric Permittivity of Single Bacterial Cells at Gigahertz Frequencies by Scanning Microwave Microscopy. ACS Nano 10 280-8

[29] Dols-Perez A, Gramse G, Calò A, Gomila G and Fumagalli L 2015 Nanoscale electric polarizability of ultrathin biolayers on insulating substrates by electrostatic force microscopy. Nanoscale 7 18327-36

[30] Esteban-Ferrer D, Edwards M A, Fumagalli L, Juárez A and Gomila G 2014 Electric Polarization Properties of Single Bacteria Measured with Electrostatic Force Microscopy ACS Nano 8 9843-9

[31] Barbet S, Popoff M, Diesinger H, Deresmes D,
Théron D and Mélin T 2014 Cross-talk artefacts in Kelvin probe force microscopy imaging: A comprehensive study J. Appl. Phys. 115144313

[32] Oliver R a 2008 Advances in AFM for the electrical characterization of semiconductors Reports Prog. Phys. 7176501

[33] Tevaarwerk E, Keppel D G, Rugheimer P, Lagally M G and Eriksson M A 2005 Quantitative analysis of electric force microscopy: The role of sample geometry Rev. Sci. Instrum. 76053707

[34] Lee M, Lee W and Prinz F B 2006 Geometric artefact suppressed surface potential measurements Nanotechnology 17 3728-33

[35] Gramse G, Gomila G and Fumagalli L 2012 Quantifying the dielectric constant of thick insulators by electrostatic force microscopy: effects of the microscopic parts of the probe. Nanotechnology 23205703

[36] Pommerville J C 2014 Fundamentals Of Microbiology (Jones \& Bartlett Learning)

[37] Wang C, Stanciu C, Ehrhardt C J and Yadavalli V K 2015 Morphological and mechanical imaging of Bacillus cereus spore formation at the nanoscale. J. Microsc. 00 1-10

[38] Hornstra L M, de Vries Y P, de Vos W M and Abee T 2006 Influence of sporulation medium composition on transcription of ger operons and the germination response of spores of Bacillus cereus ATCC 14579. Appl. Environ. Microbiol. 72 3746-9 